\title{
28 Research Square \\ What is the Role of Chest CT Scan for Pediatric Patient Diagnosis of COVID-19?
}

\section{Yaqin Zhang ( $\nabla$ zhyaqin@mail.sysu.edu.cn )}

Fifth Affiliated Hospital of Sun Yat-sen University

\section{Guangming Li}

Xiangyang Central Hospital

\section{Hongsheng Liu}

Guangzhou Women and Children's Medical Center

\section{Binghui Chen}

Fifth Affiliated Hospital of Sun Yat-sen University

\section{Zeyu Cai}

Fifth Affiliated Hospital of Sun Yat-sen University

\section{Yingqin Li}

Fifth Affiliated Hospital of Sun Yat-sen University

\section{Aamer Chughtai}

Michigan Medicine

Hong Shan

Fifth Affiliated Hospital of Sun Yat-sen University

\section{Research}

Keywords: coronavirus infections, Tomography, X-Ray Computed, follow-up studies, child

Posted Date: June 25th, 2020

DOI: https://doi.org/10.21203/rs.3.rs-35415/v1

License: (c) (1) This work is licensed under a Creative Commons Attribution 4.0 International License.

Read Full License 


\section{Abstract}

Objectives To retrospectively analyze the CT imaging features and patterns at baseline and as they evolve with time as the disease progresses or resolves in a cohort of pediatric patients affected with 2019 corona virus disease (COVID-19) pneumonia from three different cities in China.

Methods We evaluated 29 pediatric patients with COVID-19 in the authors' institution from Jan 1 to Feb 20,2020 . Cases were confirmed by laboratory test and were analyzed for epidemiological, demographic, clinical, radiological features and patterns.

Results 29 initial scans and 23 follow-up scans were obtained from 29 patients. 15(52\%) patients had been to Hubei Province and 26 (90\%) of them had close contact with the COVID-19 positive patients in 14 days prior to the illness onset. The peak severity time was 5-8 days after symptom onset. A significant difference between the number of involved segments at different time points was indicated $(p=0.019)$. Half $(52 \%)$ of the laboratory confirmed patients had no CT positive findings. Nine (31\%) of the laboratory confirmed patients had no symptoms. Six (21\%) had no CT positive findings nor symptoms. All the patients of one center $(n=6)$ whose fecal samples remained positive after the respiratory samples became negative.

Conclusion The common positive CT findings included ground-glass opacities (50\%), ground-glass opacities mixed with consolidation (36\%), peribronchial thickening (21\%), and consolidations (14\%). We recommend for pediatric patients CT should not be used as a first-line test to diagnose COVID-19.

\section{Introduction}

As of April 16th, 2020, a total of 1,991,562 confirmed 2019 coronal virus disease (COVID-19) cases and 130,885 deaths have been reported globally ${ }^{1}$. Human-to-human transmission is most likely the main transmission mode of the COVID-19 virus ${ }^{2}$. The diagnosis of COVID-19 rests mainly on the COVID-19 testing by real-time reverse-transcriptase-polymerase-chain-reaction (rRT-PCR) with specimens obtained from the respiratory tract for assay as defined by the World Health Organization (WHO $)^{3}$. In children with COVID-19 the symptoms are usually less severe than adults and present mainly with cough and fever ${ }^{4-5}$. According to the report by the Chinese Center for Disease Control and Prevention (CDC) in the confirmed cohort of 44,672 patients, 965 patients (2.2\%) aged $0-19$ years of age had case fatality rate of $0.1 \%$ (one case $)^{4}$ while aged $\geq 20$ years had case fatality rate of $2.3 \%{ }^{4}$. Most of the confirmed cases had CT scan in China. Considering the influence of the dose influence of X-ray radiation exposure get from CT scan, the role of CT scan for the pediatric patients diagnosis of COVID-19 needs to explore. We retrospectively evaluated a cohort of children under 18 years old with laboratory confirmation of COVID-19 from three different hospitals. The epidemiologic links, clinical symptom, laboratory, initial and follow-up CT imaging features are discussed in this study. We aim to evaluate the effect of CT scan for diagnosis of COVID-19 by retrospectively analyze the CT imaging features/patterns at baseline and the follow-up progress in the course of disease via a multicenter study in three different cities in China. 


\section{Materials And Methods}

This retrospective study was approved by the Institutional Review Boards our Hospital. Written consent was waived.

\section{Patients Selection}

Following the WHO COVID-19 Interim Guidance ${ }^{3}$, the enrolled patients should:

1. Children < 18 years of age;

2. Have early recognition of severe acute respiratory infection (SARI) associated with COVID-19;

3. Have positive rRT-PCR test for the COVID-19 nucleic acid;

4. Undergo CT scanning.

This study includes a total of 29 cases who were admitted for COVID-19 infection at The Fifth Affiliated Hospital of Sun Yat-sen University, Xiang Yang Central Hospital, and Guangzhou Women and Children's Medical Center. COVID-19 infection was confirmed by RT-PCR on nasopharyngeal swabs and throat swabs. All patients had serial RT-PCR results available before the time of writing. All patients had noncontrast enhanced CT scan upon admission, with follow-up CT scans available for 18 patients including 4 negative imaging patients.

\section{CT Scan and Image Analysis}

Patients were scanned using High resolution CT technique with X-ray tube voltage of $100-120 \mathrm{kV}$ and tube current of 210-250 mA. The images were reconstructed using high spatial resolution algorithm and at $1 \mathrm{~mm}$ or $2 \mathrm{~mm}$ slice thickness. The entire thorax was scanned in supine position during full suspended inspiration. Scans were interpreted on the dedicated PACS workstations using lung windows (window width, $1200 \sim 1500 \mathrm{H}$; level, -700 -1000 H) and mediastinal windows (window width, $400 \mathrm{H}$; level,40 H).

High-resolution CT (HRCT) scans were assessed for the presence and zonal distribution of ground-glass opacifications, consolidation, interlobular and intralobular interstitial septal thickening and bronchiectasis. The CT findings in the outer one third of the lung were defined as peripheral, and findings in the inner two thirds of the lung were defined as central. Consolidation was defined as an area of opacification that obscured the underlying vessels. Ground glass opacity (GGO) was defined as a hazy increase in pulmonary parenchymal attenuation without obscuring the underlying vessels. The presence of additional findings such as pleural effusions, lung fibrosis and emphysema were also evaluated.

The CT scans were reviewed by two radiologists with professional training in thoracic radiology who evaluated the pattern and distribution of the findings by consensus. The images were also evaluated for response to therapy on follow-up scans. 


\section{Real-time Reverse Transcription-Polymerase Chain Reaction (RT-PCR)}

The diagnosis of COVID-19 pneumonia performed in local CDC was based on the New Coronavirus Pneumonia Prevention and Control Program (6th edition) published by the National Health Commission of China ${ }^{5}$. All reagents and protocols used are CFDA approved. Patients with suspected SARS-CoV-2 were confirmed after two sequential positive respiratory tract sample results. In 6 patients from Fifth Affiliated Hospital of Sun Yat-sen University, fecal samples were tested. Respiratory and faecal samples were collected every 1-2 days (depending on the availability of faecal samples) until two sequential negative results were obtained.

\section{Statistical analysis}

Statistical analyses were performed using IBM SPSS Statistics Software (version 25; IBM, New York, USA) and python (version: 3.7). The difference between Number of involved segments during the disease course was analyzed by Kruskal-Wallis test. A p-value of $<0.05$ was defined as statistically significant.

\section{Results}

\section{Patients Characteristics}

A total of 29 pediatric patients with suspected SARS-CoV-2 (11 females and 18 males) were tested and confirmed with two sequential positive respiratory tract samples collected every 1-2 days. 8 patients with suspected CT image findings but the PCR resulted negative for SARS CoV-RNA. The age range was from 2 months to 17 years (mean, 7.7 years; median, 7 years). 20 patients (69\%) were $₫ 12$ years old, $9(31 \%)$ were 12-17 years old. Cough (52\%) and Fever (45\%) were the most frequent symptoms. All 6 patients'(from Fifth Affiliated Hospital of Sun Yat-sen University)fecal samples were positive for SARS CoV-RNA. The fecal samples remained positive in all of these patients after the respiratory samples became negative $(6 / 6,100 \%)$. The demographics and baseline clinical features are listed in Table 1 . Nine (31\%) of the laboratory confirmed patients had no symptoms. Six $(21 \%)$ had no CT positive findings nor symptoms. 
Table 1

Baseline clinical features of patients infected with COVID-19

\begin{tabular}{|c|c|}
\hline Features & Patients $(n=29)$ \\
\hline \multicolumn{2}{|l|}{ Age,years } \\
\hline Average(SD) & $7.7(5.0)$ \\
\hline Range & $0.2-17$ \\
\hline$\nabla 12$ & $20(69 \%)$ \\
\hline $12-17$ & $9(31 \%)$ \\
\hline \multicolumn{2}{|l|}{ Sex } \\
\hline Female & $11(38 \%)$ \\
\hline Male & $18(62 \%)$ \\
\hline \multicolumn{2}{|l|}{ Epidemiological history } \\
\hline Wuhan travel/residence history & $5(17 \%)$ \\
\hline Close contact with the COVID-19 patients & $26(90 \%)$ \\
\hline Admission to intensive care unit & $0(0 \%)$ \\
\hline \multicolumn{2}{|l|}{ Clinical Outcome } \\
\hline Discharged & $29(100 \%)$ \\
\hline Died & $0(0 \%)$ \\
\hline Symptom & $20(69 \%)$ \\
\hline Fever & $15(52 \%)$ \\
\hline \multicolumn{2}{|l|}{ Highest temperature, ${ }^{\circ} \mathrm{C}$} \\
\hline 凶37.3 & $16(55 \%)$ \\
\hline $37.3-38.0$ & $6(21 \%)$ \\
\hline $38.1-39.0$ & $6(21 \%)$ \\
\hline$\varangle 39.0$ & $1(3 \%)$ \\
\hline Cough & $15(52 \%)$ \\
\hline Runny nose & $1(3 \%)$ \\
\hline Sore throat & $1(3 \%)$ \\
\hline Headache & $1(3 \%)$ \\
\hline No symptom & $9(31 \%)$ \\
\hline
\end{tabular}




\begin{tabular}{|ll|}
\hline Features & Patients $(\mathrm{n}=29)$ \\
\hline Laboratory findings & \\
\hline Lymphocyte count $\left(\times 10^{9} / \mathrm{L}\right)$ & 3.07 \\
\hline White blood cell count $\left(\times 10^{9} / \mathrm{L}\right)$ & 7.14 \\
\hline Neutrophil count $\left(\times 10^{9} / \mathrm{L}\right)$ & 3.62 \\
\hline
\end{tabular}

According to the WHO COVID-19 Interim Guidance ${ }^{3}$ and the Sixth Edition Guideline of 2019-nCoV published by National Health Commission of China ${ }^{6}$, we divided the confirmed cases into 2 groups according to the clinical symptoms and imaging findings: 15 patients in the 'imaging negative group'. In this group we included the patients whose CT imaging had no positive findings but had laboratory confirmed positive for COVID-19. The remainder of 14 cases were categorized as mild group. There were no patients in the severe and critical groups.

There were 5 (17\%) patients who had been to Hubei Province and $26(90 \%)$ of them had close contact with the COVID-19 positive patients. All patients denied the history of visiting the Huanan Seafood Wholesale Market in Wuhan in January. Some patients had history of preexisting conditions such as asthma $(n=1)$, congenital heart disease $(n=1)$, hernia after surgery $(n=2)$. There were no deaths among these patients.

\section{CT imaging Features}

All of the 29 patients had the initial scan at admission as the baseline. The average days from illness onset till the initial scan were 3.2 days (range: $0-12$ days).

The initial high-resolution CT scan was performed $1.8 \pm 1.3$ days after admission to the hospital.

In the Mild Group ( $\mathrm{n}=14$ ), the lesions were predominantly peripheral in $71 \%$ of patients and both peripheral and central in $29 \% .92 \%$ of patients had lower lobe involvement while only 1 patient had single upper lobe lesion.

The details of the CT findings are summarized in Table 2. Ground-glass opacity (GGO) $(n=7)$ was the most common CT finding in the initial scan after admission followed by GGO mixed with consolidation $(n=5)$, peribronchial thickening $(n=3)$, consolidation $(n=2)$, air bronchogram $(n=1)$, interlobular and intralobular septal thickening $(n=1)$ (Fig. 1). 15 patients were categorized as imaging negative with the CT scan showing no pulmonary parenchymal abnormalities. 
Table 2

CT imaging features of patients infected with COVID-19

\begin{tabular}{|c|c|c|c|}
\hline & Baseline & Second scan & Third scan \\
\hline Virus pneumonia cases & 14 & 14 & 14 \\
\hline Virus pneumonia cases with CT scan & 21 & 9 & 3 \\
\hline Imaging negative cases & 15 & 15 & 15 \\
\hline Imaging negative cases with CT scan & 15 & 9 & 2 \\
\hline Whole cohort had CT scan & 29 & 18 & 5 \\
\hline Whole cohort & 29 & 29 & 29 \\
\hline Average involved lobes & 1.7 & 1.8 & 1.7 \\
\hline Average involved segments & 2.5 & 2.6 & 1.7 \\
\hline \multicolumn{4}{|l|}{ Area } \\
\hline Central & 0 & 0 & 0 \\
\hline Peripheral & 10 & 5 & 2 \\
\hline both & 4 & 3 & 3 \\
\hline \multicolumn{4}{|l|}{ Side } \\
\hline Right & 4 & 2 & 2 \\
\hline Left & 6 & 3 & 1 \\
\hline Both & 4 & 3 & 2 \\
\hline \multicolumn{4}{|l|}{ Features } \\
\hline Ground Glass & $7(50 \%)$ & $5(56 \%)$ & $4(80 \%)$ \\
\hline Consolidation & $2(14 \%)$ & $1(11 \%)$ & $0(0 \%)$ \\
\hline Mixed GGO and consolidation & $5(36 \%)$ & $3(33 \%))$ & $1(20 \%)$ \\
\hline Scar & 0 & 0 & 0 \\
\hline Interlobular septal & $1(8 \%)$ & 0 & 0 \\
\hline Intralobular septal & $1(8 \%)$ & 0 & 0 \\
\hline Bronchiectasis & 0 & 0 & 0 \\
\hline Peribranchial thickening & $3(21 \%)$ & $2(11 \%)$ & $1(17 \%)$ \\
\hline Air bronchogram & $1(7 \%)$ & $2(11 \%)$ & 0 \\
\hline
\end{tabular}




\begin{tabular}{|llll|}
\hline & Baseline & Second scan & Third scan \\
\hline Plural effusion & 0 & 0 & 0 \\
\hline
\end{tabular}

We classified the CT imaging patterns of the COVID-19 infection as follows.

Type A. Sub-pleural patchy consolidation/consolidation mixed with GGO/only GGO (9, 31\%) in single lobe or both lungs (Fig. 2)

Type B. Peribronchial wall thickening with or without GGO (3, \%) (Fig. 3)

Type C. Large area of consolidation with or without GGO (2, \%) (Fig. 4)

The CT pattern of the patients between 12-18 years of age are belonged to the type A $(n=5,45 \%), 1$ belonged to the type $C(9 \%)$, and $45 \%$ belonged to the imaging negative group. Among the children between 0 to 12 years old $(n=20), 3$ belonged to the type $B, 6$ belonged to the type $A, 1$ belonged to the type $C, 10$ belonged to the imaging negative group. Comparing with Teenage patients, younger children presented with larger variation of image patterns.

Follow-up second and third CT scans were obtained after admission depending on the clinical condition of the patients. 18 patients had a second scan, and 5 patients had a third scan. On the follow-up CT scans, the patchy GGO or GGO mixed with patchy consolidation or centrilobular nodules with bronchial wall thickening was mostly resolved or completely resolved on the second scan; while the Type $C$ consolidation is relatively slower be resolved such as: an 14-year old female which had a $16 * 15 \mathrm{~mm}$ subpleural consolidation in the left lower lobe. 16 days later the follow-up CT scan showed the lesion partly resolved and turned to be GGO.

\section{Time Course of CT image Changes}

From symptom onset the number of involved segments of bilateral lungs are illustrated in Fig. 5. The highest number of segments recorded was 6 . The number of involved segments changed over time (Fig. $5, p=0.019$ ). Peak severity was reached at 5-8 days, at which the involved segments was ranged from $1-6$, and the average was 2.8 .

\section{CT Correlation with RT-PCR}

The RT-PCR results were used as golden standard. 15 patients had no CT image abnormalities. One patient was normal in the initial scan and had ground-glass opacity in the second scan. The baseline CT scan sensitivity was $45 \%(13 / 29)$.

\section{Discussion}


In this study we evaluated a cohort of 29 pediatric patients with laboratory confirmed COVID-19 infection. All cases had the history of travel to or residence in Wuhan or other cities of Hubei Province or close contact history with confirmed patients. The most common CT features are (1) Ground-glass opacity; (2) Ground-glass opacity mixed with consolidation; (3) Centrilobular nodules with bronchial wall thickening. And the most common CT pattern in the entire cohort is Type A-Sub-pleural patchy consolidation/consolidation mixed with GGO/only GGO. Peak severity which reflected by number of involved segments on CT was at 5-8 days after symptom onset. And the peak severity of adults was reported at 10-12 days on CXR and 6-11 days on $\mathrm{CT}^{7-10}$

We noted the two distinct imaging patterns among the children we studied. Teenage $(\geq 10$ years $)$ patients mainly presented with Type A patchy consolidation/consolidation mixed with GGO/GGO in single or both lungs or normal, whereas the younger children presented mainly with all the 3 types including Type $B$ centrilobular nodules with bronchial wall thickening and Type $C$ large area of consolidation. The clinical course of patients with Types A, B was much milder and shorter compared with the patients with $\mathrm{C}$. The younger children with Type $C$ pattern who had large area of consolidation, the clinical course was more severe and the lesions resolved more slowly. This is different from the previous reports about children with SARS. In that report from Hong Kong ${ }^{11} 44$ younger children ( $\leq 12$ years) had much milder and shorter clinical course compared to the teenagers (12-18ys). There are individual differences in the course of the disease. Looking at the temporal change in the imaging patterns from the time of admission till discharge, we recommend the follow-up CT scans to be obtained about 10-15 days after the initial scan in Types $A$ and $B$ patients. In Type $C$ patients, we suggest the follow-up scan could be obtained at least 1 month later or even longer. Imaging negative group occupied about $52 \%$ of the included patients. These pediatric patients with normal CT findings may do not need follow-up scan any more if the symptomatic treatment is effective.

The CT findings of COVID-19 pneumonia are similar as RSV pneumonia, HPIV pneumonia, and HMPV pneumonia ${ }^{12}$. These viral infections usually appear as multifocal patchy consolidation with GGO. Centrilobular nodules with bronchial wall thickening are also noted. Large area of consolidation with or without GGO are uncommon but more extensive when present. Different from adult patients with COVID19 infection, the interlobular and intralobular septal thickening $(25-53 \%)^{8-10,13}$ is less common in children (our study $8 \%$ ) and the percentage of imaging negative patients is relatively much higher (our study $52 \%$ vs $\left.19 \%{ }^{10}\right)$.

Radiologists should familiarize themselves with the CT appearance of COVID-19 infection in order to be able to identify findings consistent with infection in patients imaged for other reasons. The analysis of CT patterns/features could help the clinicians to evaluate the outcome of the infection and select the suitable follow-up scan time. In our laboratory confirmed cohort, 9 patients were asymptomatic. 16 of these patients had no positive CT imaging findings. 6 patients were asymptomatic but had positive CT imaging findings. A normal chest CT does not mean a person does not have COVID-19 infection - and an abnormal CT is not specific for COVID-19 diagnosis ${ }^{14}$. We suggest laboratory confirmed cases should be 
isolated regardless of being asymptomatic. We also recommend for pediatric patients CT should not be used as a first-line test to diagnose COVID-19 as the ACR Recommendations ${ }^{14}$.

Besides, all of the included 6 pediatric patients from one medical center had positive SARS-CoV-2 RNA fecal samples (100\%). Xi Huang et $\mathrm{al}^{15}$ evaluated the infection in patients from this center and found (55\%) of 74 patients with fecal samples were positive for SARS-CoV-2 RNA. Corman et al ${ }^{16}$ found MERSCoV RNA in $14.6 \%$ of stool samples from patients with MERS-CoV. In a recently published article, the authors report a prolonged detection of viral RNA for 20 days or more commonly seen in patients with SARS-CoV-2 infection similar to MERS-CoV or SARS-CoV infections ${ }^{17-19}$. In our study, we agree with obtaining posterior oropharyngeal saliva samples and stool samples for testing with real-time RT-PCR. This is highly recommended after the clearance of viral RNA in a patient's respiratory samples, especially in the pediatric patients ${ }^{15}$. This may potentially help in controlling oral-fecal transmission in confined premises such as hostels, dormitories, trains, buses, and cruise ships.

There are few limitations in our study. Firstly, the number of cases in this study is small. It is important to note that the entire range of imaging features may not have been fully demonstrated during the time course since the emergence of COVID-19 outbreak. Secondly, only in one medical center the fecal samples were tested for SARS-CoV-2 RNA. Future studies should involve a larger patient population. Finally, we do not have the histopathologic evidence to reflect the imaging changes throughout the entire course of infection.

\section{Conclusion}

In summary, the most common CT imaging features of COVID-19 infection in pediatric patients are predominantly: 1 . Ground-glass opacity. 2. Ground-glass opacity mixed with consolidation. 3. Peribronchial thickening. About half $(52 \%)$ of the laboratory confirmed patients had no CT positive findings. We recommend for pediatric patients CT should not be used as a first-line test to diagnose COVID-19.

\section{Declarations}

\section{Ethics approval and consent to participate}

This retrospective study was approved by the Institutional Review Boards of The Fifth Affiliated Hospital of Sun Yat-sen University. Written consent was waived

\section{Consent for publication}

All presentations of case reports must have consent for publication. 


\section{Availability of data and materials}

The datasets used and/or analysed during the current study are available from the corresponding author on reasonable request.

All data generated or analysed during this study are included in this published article [and its supplementary information files].

\section{Competing interests}

The authors declare that they have no competing interests

\section{Funding}

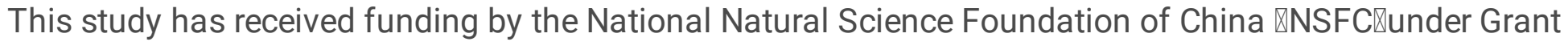
81801809, the Guangdong Basic and Applied Basic Research Foundation 2020A1515010572 and the Guangdong Province Key Laboratory of Computational Science Open Grant 2018009.

\section{Authors' contributions}

Dr Zhang conceptualized and designed the study, drafted the initial manuscript, and reviewed and revised the manuscript.

Drs Li, and Liu designed the data collection instruments, collected data, carried out the initial analyses, and reviewed and revised the manuscript.

Drs Chen and Cai coordinated and supervised data collection.

Dr Li organized collected data.

Dr Chughtai critically reviewed the manuscript for important intellectual content, reviewed and revised the manuscript.

Dr Shan reviewed and revised the manuscript.

All authors approved the final manuscript as submitted and agree to be accountable for all aspects of the work.

\section{Acknowledgements}


We would like to thank the doctors in China that have dedicated their time and efforts to treat COVID-19 patients and protect the health of all Chinese during this time.

\section{References}

1. WHO. Coronavirus disease (COVID-2019) situation reports. https://www.who.int/docs/defaultsource/coronaviruse/situation-reports/20200229-sitrep-40-covid-19.pdf?sfvrsn=7203e653_2

2. Rothan H A, Byrareddy S N. The epidemiology and pathogenesis of coronavirus disease (COVID-19) outbreak. Journal of Autoimmunity, 2020: 102433.

3. WHO. Clinical management of severe acute respiratory infection when novel coronavirus (nCoV) infection is suspected. Available at: https://www.who.int/publications-detail/clinical-managementof-severe-acute-respiratory-infection-when-novel-coronavirus-(ncov)-infection-is-suspected. Accessed Jan 28, 2020.

4. Cai J, Xu J, Lin D, Yang Z, Xu L, Qu Z et al. A case series of children with 2019 novel coronavirus infection: clinical and epidemiological features. Clin Infect Dis. 2020. Epub 2020/03/01. doi: 10.1093/cid/ciaa198. PubMed PMID: 32112072.

5. Xia W, Shao J, Guo Y, Peng X, Li Z, Hu D. Clinical and CT features in pediatric patients with COVID-19 infection: different points from adults. Pediatr Pulmonol. 2020. Epub 2020/03/07. doi: 10.1002/ppul.24718. PubMed PMID: 32134205.

6. http://www.nhc.gov.cn/jkj/s3577/202003/4856d5b0458141fa9f376853224d41d7.shtml

7. Novel coronavirus pneumonia emergency response epidemiology team. The epidemiological characteristics of an outbreak of 2019 novel coronavirus diseases (COVID-19) in China. Zhonghua Liu Xing Bing Xue Za Zhi, 2020. Published online Feb 17, 2020 https://doi.org/ 10.3760/cma.j.issn.0254-6450.2020.02.003

8. Wong H Y F, Lam H Y S, Fong A H T, et al. Frequency and Distribution of Chest Radiographic Findings in COVID-19 Positive Patients. Radiology, 2020: 201160.

9. Pan F, Ye T, Sun P, et al. Time course of lung changes on chest CT during recovery from 2019 novel coronavirus (COVID-19) pneumonia. Radiology, 2020: 200370.

10. Wang Y, Dong C, Hu Y, et al. Temporal changes of CT findings in 90 patients with COVID-19 pneumonia: a longitudinal study[J]. Radiology, 2020: 200843.

11. Leung C, Kwan Y, Ko P, et al. Severe acute respiratory syndrome among children[J]. Pediatrics, 2004, 113(6): e535-e543.

12. Koo H J, Lim S, Choe J, et al. Radiographic and CT features of viral pneumonia[J]. Radiographics, 2018, 38(3): 719-739.

13. Chung M, Bernheim A, Mei X, et al. CT imaging features of 2019 novel coronavirus (2019-nCoV). Radiology, 2020: 200230.

14. https://www.acr.org/Advocacy-and-Economics/ACR-Position-Statements/Recommendations-forChest-Radiography-and-CT-for-Suspected-COVID19-Infection 
15. Wu Y, Guo C, Tang L, et al. Prolonged presence of SARS-CoV-2 viral RNA in faecal samples. The Lancet Gastroenterology \& Hepatology, 2020.

16. Corman VM, Albarrak AM, Omrani AS, et al. Viral shedding and antibody response in 37 patients with Middle East respiratory syndrome coronavirus infection. Clin Infect Dis 2016; 62: 477-83.

17. To K K-W, Tsang O T-Y, Leung W-S, et al. Temporal profiles of viral load in posterior oropharyngeal saliva samples and serum antibody responses during infection by SARS-CoV-2: an observational cohort study. The Lancet Infectious Diseases, 2020.

18. Peiris JSM, Chu CM, Cheng VCC, et al. Clinical progression and viral load in a community outbreak of coronavirus-associated SARS pneumonia: a prospective study. Lancet 2003; 361: 1767-72.

19. Oh MD, Park WB, Choe PG, et al. Viral load kinetics of MERS coronavirus infection. N Engl J Med 2016; 375: 1303-05.

\section{Figures}

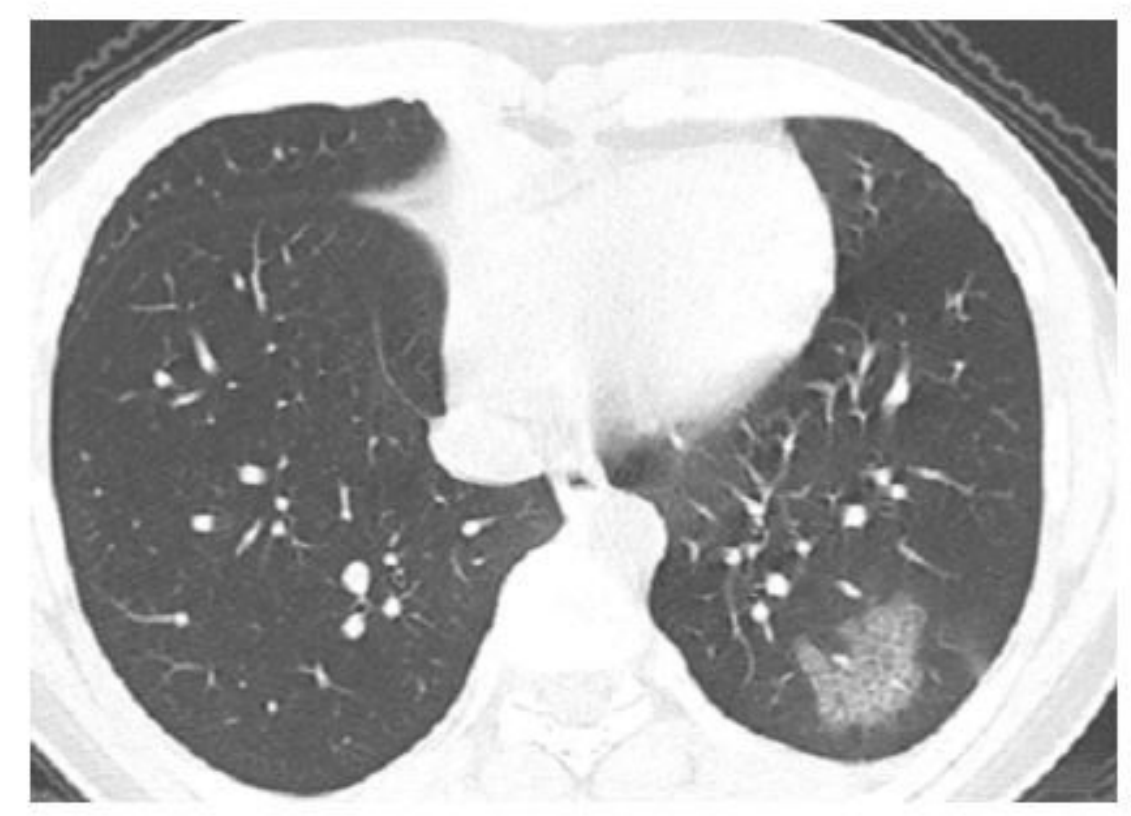

\section{Figure 1}

Chest image of a COVID-19 pneumonia patient with septal thickening on non-contrast CT A 14-year-old male diagnosed with COVID-19 had the close contract history with laboratory confirmed patients. High resolution CT scan demonstrated ground-glass opacity with thickening of interlobular and intralobular septal thickening (crazy paving-pattern) in the subpleural lateral basal segment of left lower lobe. 


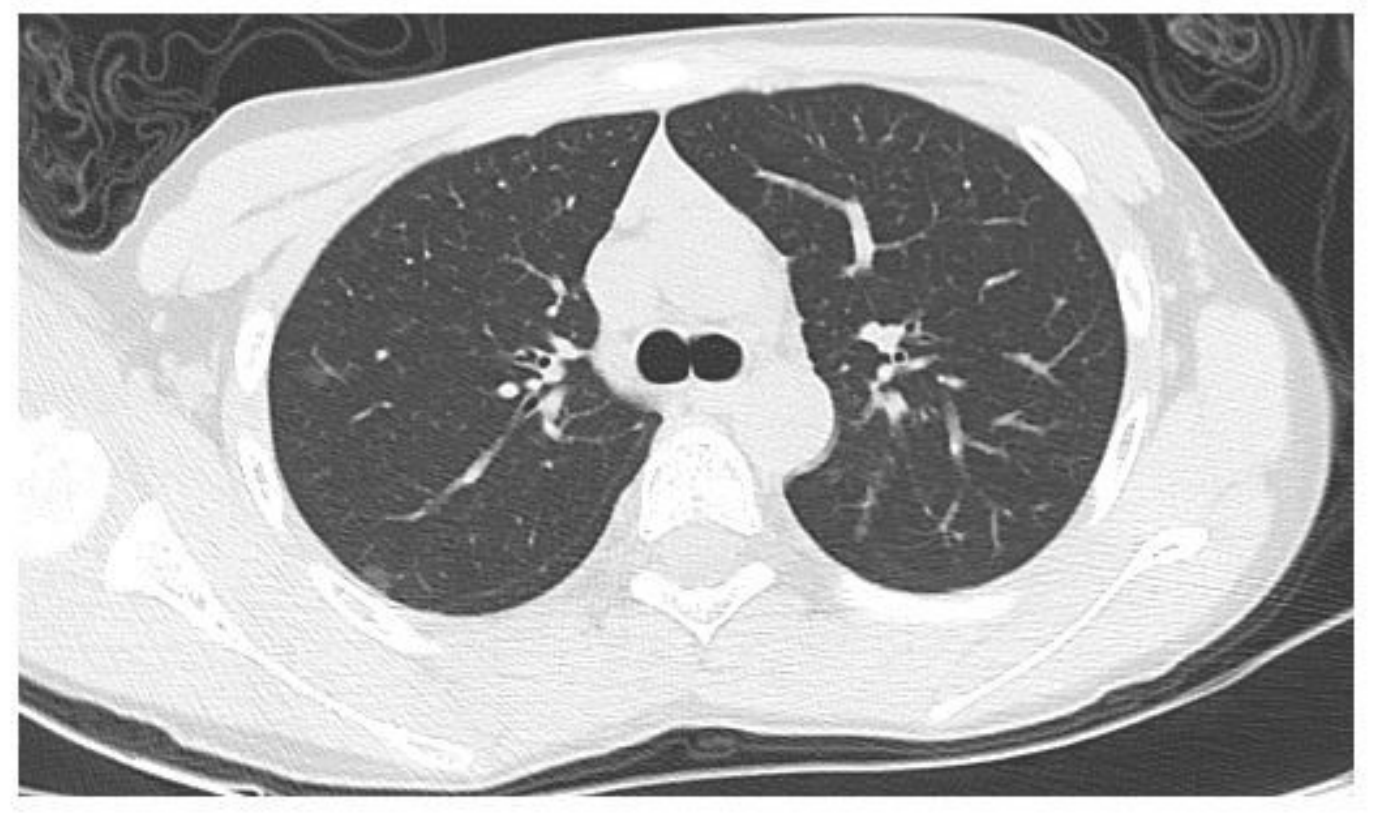

\section{Figure 2}

Chest image of a COVID-19 pneumonia patient with Type A CT image pattern A 11-year-old male without any symptoms which had the travel history to Wuhan on Jan 20 was diagnosed with COVID-19 on Feb 14. The non-contrast CT images showed tiny subpleural patchy ground-glass opacification was located in the right upper lobe.

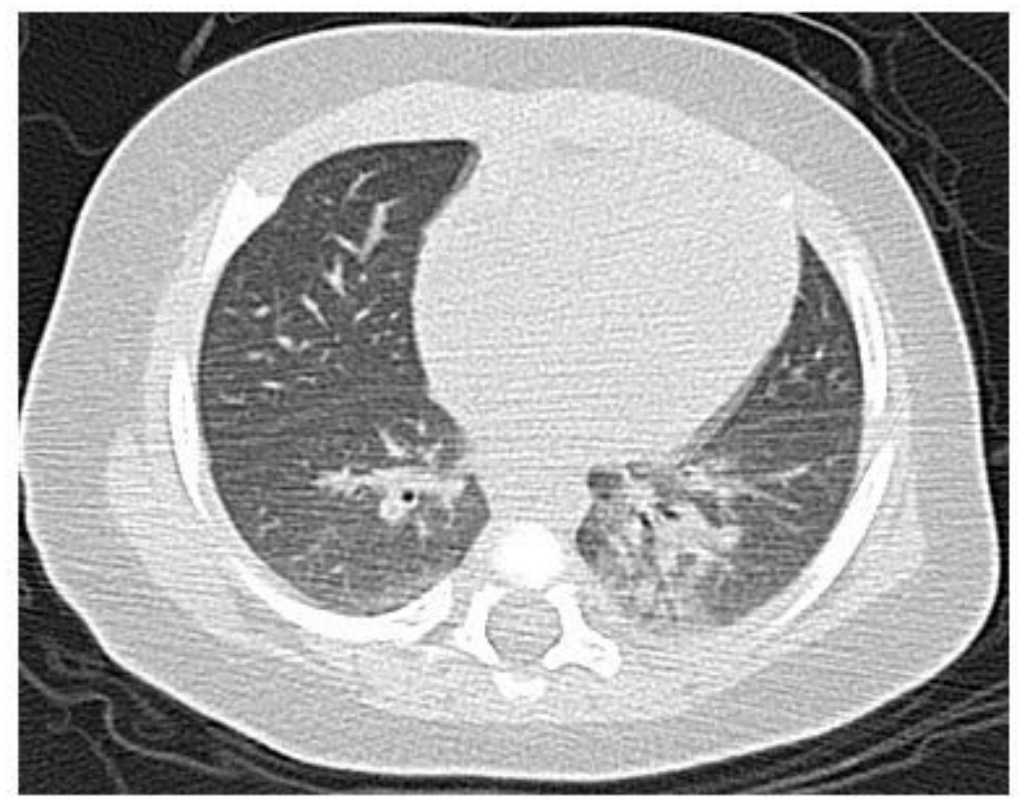

Figure 3 
Chest image of a COVID-19 pneumonia patient with Type B CT image pattern Axial non-contrast enhanced CT images of a 2-month old female infant diagnosed with COVID-19 with recent travel history to Wuhan. She presented with a 1-day history of cough. The baseline CT demonstrates centrilobular nodules with bronchial wall thickening in basal segments of left lower lobe.

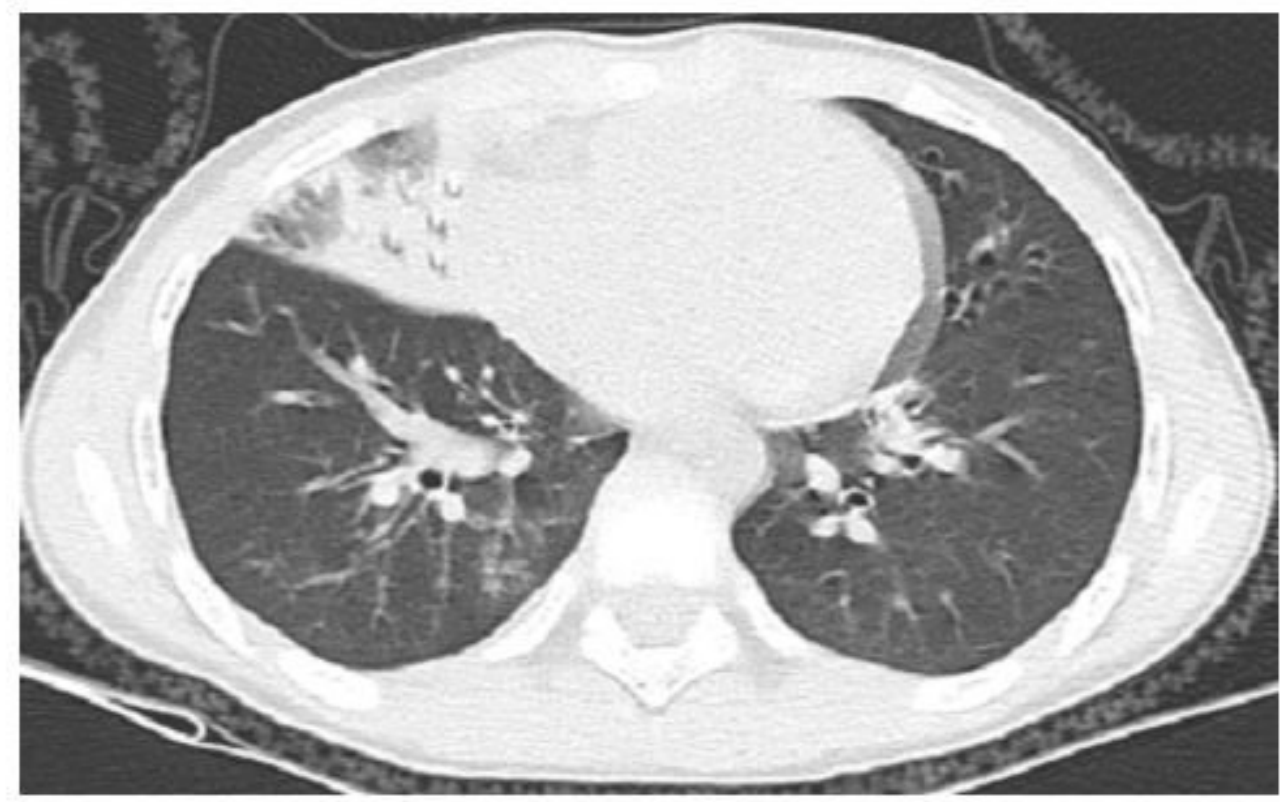

\section{Figure 4}

Chest image of a COVID-19 pneumonia patient with Type C CT image pattern A 7-year-old female presented with a 1-day history of fever $₫ 37.7^{\circ} \mathrm{C}$ ) and 3-day history of cough. She had the close contract history with laboratory confirmed patient. Non-contrast CT showed large area of consolidation in the right middle lobe and ground-glass opacity in the basal segment of right lower lobe. 


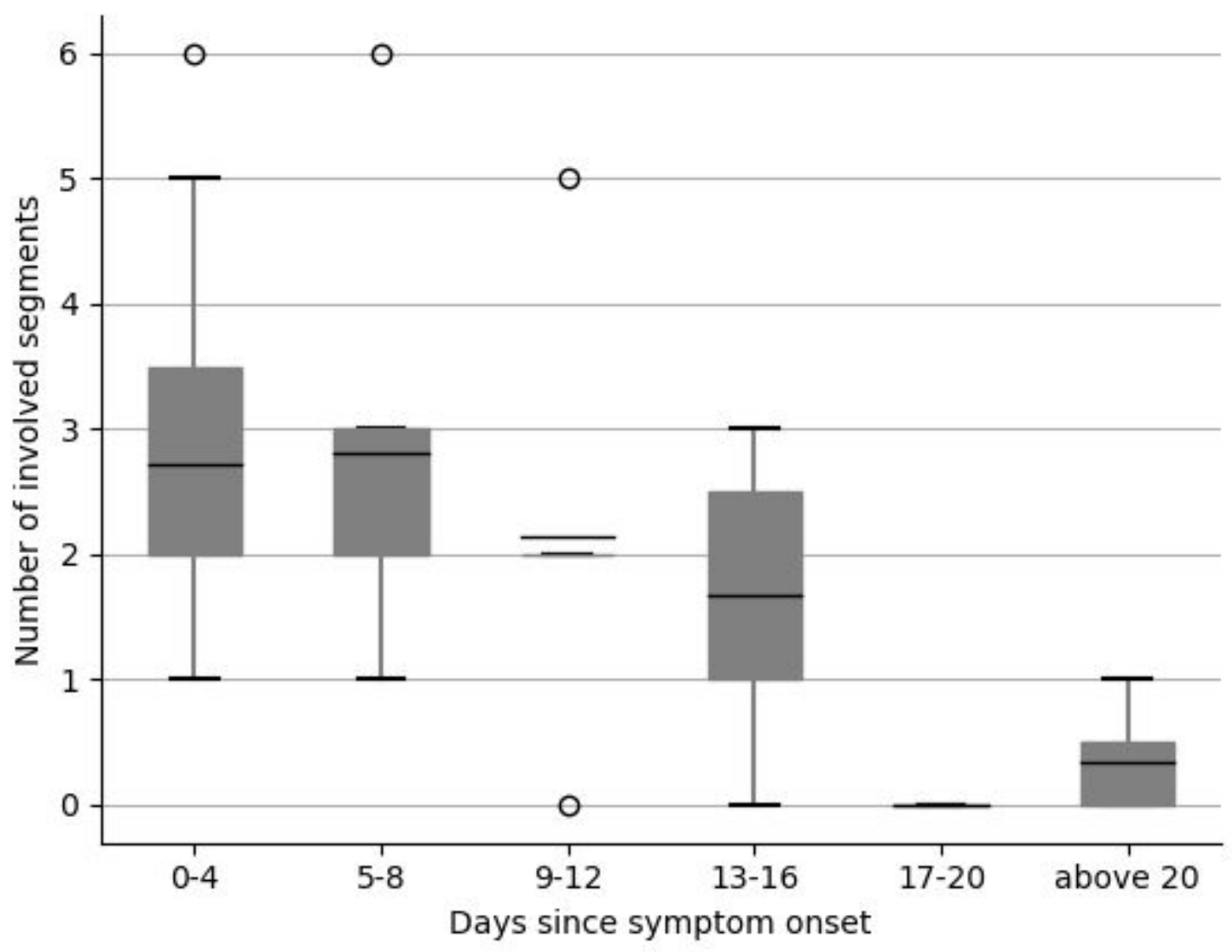

Figure 5

Change in COVID-19 non-contrast CT involved segments with duration since symptom onset. The number of the involvement by consolidation or GGO ranged of 0-6 was assigned to bilateral lungs. Kruskal-Wallis test $(p=0.019)$ indicated a significant difference between the number of involved segments at different time points.

\section{Supplementary Files}

This is a list of supplementary files associated with this preprint. Click to download.

- TitlePage..docx 